\title{
Dextromethorphan attenuated the higher vulnerability to inflammatory thermal hyperalgesia caused by prenatal morphine exposure in rat offspring
}

\author{
Pao-Luh Tao ${ }^{1}$, Chien-Fang Chen ${ }^{2}$ and Eagle Yi-Kung Huang ${ }^{2^{*}}$
}

\begin{abstract}
Background: Co-administration of dextromethorphan (DM) with morphine during pregnancy and throughout lactation has been found to reduce morphine physical dependence and tolerance in rat offspring. No evidence was presented, however, for the effect of DM co-administered with morphine during pregnancy on inflammatory hyperalgesia in morphine-exposed offspring. Therefore, we attempt to investigate the possible effect of prenatal morphine exposure on the vulnerability to hyperalgesia and the possible therapeutic effect of DM in the present study.

Methods: Fifty $\mu \mathrm{l}$ of carrageenan $(20 \mathrm{mg} / \mathrm{ml})$ was injected subcutaneously into the plantar surface of the right hind paw in p18 rats to induce hyperalgesia. Mean paw withdrawal latency was measured in the plantar test to index the severity of hyperalgesia. Using Western blotting and RT-PCR, the quantitative analyses of NMDA receptor NR1 and NR2B subunits were performed in spinal cords from different groups of animals.

Results: In the carrageenan-induced hyperalgesia model, rat offspring passively exposed to morphine developed a severe hyperalgesia on postnatal day 18 (p18), which also had a more rapid time course than those in the controls. Co-administration of DM with morphine in the dams prevented this adverse effect of morphine in the offspring rats. Western blot and RT-PCR analysis showed that the levels of protein and mRNA of NMDA receptor NR1 and NR2B subunits were significantly higher in the lumbar spinal cords of rats (p14) exposed to prenatal morphine; the co-administration of DM could reverse the effect of morphine on NR1 and attenuate the effect on NR2B.
\end{abstract}

Conclusions: Thus, DM may have a great potential in the prevention of higher vulnerability to inflammatory thermal hyperalgesia in the offspring of morphine-addicted mothers.

\section{Background}

Growth retardation, delayed motor development and behavior abnormalities have been proposed in offspring of heroin-addicted mothers [1]. Infants passively exposed to morphine through their addicted mothers easily developed morphine withdrawal syndrome after birth, and even needed intensive care [2-4]. In our previous studies, we observed that many adverse effects caused by prenatal exposure of morphine could be prevented by the co-

\footnotetext{
* Correspondence: eyh58@mail.ndmctsgh.edu.tw

${ }^{2}$ Department of Pharmacology, National Defense Medical Center, Taipei, NeiHu 114, Taiwan

Full list of author information is available at the end of the article
}

administration of dextromethorphan (DM) in morphinedependent rat dams $[5,6]$. However, the possible impacts of prenatal exposure of morphine on the vulnerability to hyperalgesia have never been examined. In humans, the liability to inflammatory hyperalgesia is often affected by acquired physical conditions and social factors in offspring from morphine-addicted mother [7]. Thus, we attempted to investigate the possible effects of prenatal exposure to morphine on the vulnerability to hyperalgesia in a rat model. In addition, the possible protective effect of the co-administered DM was also tested.

Being a non-competitive antagonist at the glutamatergic NMDA receptors, DM is thought to exert many of

\section{Biomed Central}


its pharmacological actions through the blockade of NMDA receptor [8], although DM was reported to act at the other targets (e.g. nicotinic $\alpha 3 \beta 4$ receptors and sigma receptors) as well $[9,10]$. Activation of the NMDA receptors has been implicated in the development of inflammatory hyperalgesia and the regulation of rewarding-related mesolimbic pathway in many reports [11-13]. Therefore, we speculated that the prenatal administration of morphine and DM to the dams may affect the development of the neural systems which will be functionally correlated with hyperalgesia in the offspring. In order to investigate the vulnerability to hyperalgesia, we used a plantar test in rats with intraplantar carrageenan-induced inflammatory hyperalgesia to perform quantitative verification. These behavioral experiments were carried out on the rats which were the offspring from the morphine-dependent dams.

In the present study, we first demonstrated that the prenatal exposure of morphine could increase the vulnerability to inflammatory hyperalgesia in the offspring. Our biochemical results showed a clear increase of the NR1 and NR2B subunits of NMDA receptors in the spinal cord from the offspring (18 days old; p18) of morphine-treated mother. This could provide some neural developmental evidence which may be related to the higher vulnerability to inflammatory hyperalgesia. This adverse effect of prenatal morphine exposure could be prevented by the co-administration of DM in the dams. Overall, our results highlighted the possible adverse effect of prenatal morphine exposure that is seldom noticed. DM may also have a great therapeutic potential in the prevention of the adverse effect.

\section{Materials and methods}

Animals

Adult female Sprague-Dawley rats were purchased from the National Experimental Animal Centre, Taipei, Taiwan. The animals were housed one or two in a cage, in a room maintained at a temperature of $23 \pm 2^{\circ} \mathrm{C}$ with a $12 \mathrm{~h}$ lightdark cycle. Food and water were available ad libitum throughout the experiment. Rats were randomly separated into four groups. Rats received subcutaneous (s.c.) injection of saline (Control group), morphine (M group), morphine + dextromethorphan $(\mathrm{M}+\mathrm{DM}$ group) and dextromethorphan (DM group) twice per day (9 AM and $5 \mathrm{PM}$ ) and progressively increased with $1 \mathrm{mg} / \mathrm{kg}$ at 7-day intervals from a beginning dose of $2 \mathrm{mg} / \mathrm{kg}$ for both morphine and dextromethorphan. The rats were mated on day 8 and the drug administration was continued during pregnancy. After rat offspring were born, the dams were housed separately in individual cages and the injections of drugs into the dams were stopped. Four groups of neonatal rats of either sex aged 18 days (p18) were used for the plantar test. P14 rats were used for Western blot and RT-
PCR analysis. The care of animals was carried out in accordance with institutional and international standards (Principles of Laboratory Animal Care, National Institutes of Health), and the protocol was approved by the Institutional Animal Care and Use Committee of National Defense Medical Center, Taiwan, R.O.C.

\section{Determination of carrageenan-induced thermal hyperalgesia}

P18 rats from four groups were used for plantar tests. Fifty $\mu \mathrm{l}$ of carrageenan $(20 \mathrm{mg} / \mathrm{ml})$ was injected subcutaneously via a $28-\mathrm{G}$ needle into the plantar surface of the right hind paw. A plantar analgesiometer (7370, Ugo Basile, Italy) was used to index thermal hyperalgesia [14]. Mean paw withdrawal latency in response to the stimulus from a focused beam of I.R. light served as the measure of thermal nociception. Cut-off time of the paw withdrawal latency was set at $10 \mathrm{sec}$ to prevent thermal injury. Animals were all injected with carrageenan on the right hind paw that was subjected to the determination of paw withdrawal latency. The paw withdrawal latencies were measured before carrageenan injection $(0$ hour), and at the time points after carrageenan injection: $3,6,9,12,24,48$ hours. The paw withdrawal latency of each rat was tested for three times at each time point. Two closer values of latencies were selected and averaged as the final data to be used. The paw withdrawal latencies were calculated and converted to the percentage of the basal latency for comparison.

\section{Western blot analysis}

P14 rats from four groups were sacrificed by decapitation, and their lumbar (L1 to L6) spinal cords were quickly dissected. These tissues were immediately frozen in liquid nitrogen and kept at $-80^{\circ} \mathrm{C}$ until use. The tissues were disrupted by homogenization on ice in lysis buffer [Tris-HCl $0.05 \mathrm{M}$, EDTA $5 \mathrm{mM}, \mathrm{NaCl} 0.15 \mathrm{M}$, Triton X-100 1\%, aprotinin $0.5 \mu \mathrm{g} / \mathrm{ml}$, leupeptin $0.5 \mu \mathrm{g} /$ $\mathrm{ml}$, phenylmethanesulfonyl fluoride (PMSF) $30 \mu \mathrm{g} / \mathrm{ml}$, 1,4-dithiothreitol (DTT) $5 \mathrm{mM}$ ]. Tissue lysates were obtained by first centrifugation at $1,000 \times \mathrm{g}$ for $10 \mathrm{~min}$, followed by the second centrifugation of the previous supernatant at $35,000 \times \mathrm{g}$ for $30 \mathrm{~min}$ at $4^{\circ} \mathrm{C}$. Protein concentrations were estimated by the BCA protein assay (Pierce, U.S.A.) using bovine serum albumin (BSA) as standards. For Western blot analysis, $25 \mu \mathrm{g}$ protein of the lysates was dissolved in sample buffer (Tris $0.2 \mathrm{M}$, SDS $0.8 \%$, glycerol 5\%, DTT $3.1 \mathrm{mg} / \mathrm{ml}$, bromophenol blue $0.04 \mathrm{mg} / \mathrm{ml}$ ), boiled for $5 \mathrm{~min}$, and subjected to SDS-PAGE (10\% polyacrylamide). The proteins on the gel were transferred to a polyvinylidene fluoride (PVDF) membrane (FluoroTrans W membrane, pore size: 0.2 $\mu \mathrm{m}$, PALL Life Sciences, U.S.A.) using a GENIE electrophoretic transfer apparatus (Idea Scientific, U.S.A.). The 
membranes were incubated with first antibody [1:1K; anti-NMDA NR1 monoclonal antibody, anti-NMDA NR2B polyclonal antibody (Novus Biologicals, U.S.A.), and anti- $\beta$-actin monoclonal antibody (Sigma, U.S.A.)] at $4^{\circ} \mathrm{C}$ over night, and then incubated with secondary antibody [1:2K; anti-rabbit IgG HRP conjugated antibody and anti-mouse IgG HRP conjugated antibody (Alpha Diagnostic, U.S.A.)] for one hour. The bands of proteins were revealed by ECL Western blotting detection kit (Amersham Biosciences, U.K.) and visualized on an X-ray film. Using Kodak Digital Science 1D image analysis software, the optical density of each band was analyzed. The value was calculated as the ratio of the density of corresponding $\beta$-actin bands. These ratios were then normalized with the mean ratio obtained from the control group, which was set as $100 \%$.

\section{Reverse transcription polymerase chain reaction (RT-PCR)} Tissue samples were obtained from the lumbar spinal cords of the p14 rats from four groups. These P14 rats were sacrificed by decapitation, and their lumbar (L1 to L6) spinal cords were quickly dissected. These tissues were immediately frozen in liquid nitrogen and kept at $-80^{\circ} \mathrm{C}$. Total RNA of the samples was extracted with EZ10 Spin Column Total RNA MiniPreps Super Kit (Bio Basic Inc., Canada). One $\mu$ g of total RNA was used per PCR condition. Using the One-Step RT PCR kit (GeneMark, Taiwan, R.O.C.), forty PCR cycles were performed and the products were subjected to agarose gel electrophoresis. Primers used were (5'-3', sense/antisense): TGGGACACGGCTCTGGAAG/TAGGCGGGTGGCTAACTA for NR1, AGCCAAGAGGAGGAAACAGC/ ACCTCCACTGACCGAATCTC for NR2B). Using Kodak Digital Science 1D image analysis software, quantitative analysis was performed after scanning of the ethidium bromide-stained agarose gel pictures. The method of quantification was similar to that used in Western blotting, but the bands of GAPDH were used as the internal controls.

\section{Statistical analysis}

The data were all expressed as means \pm SEM. One-way ANOVA followed by Newman-Keuls test was employed to examine the statistical significance of the difference between groups.

\section{Results}

Chronic morphine administration of the dams caused a higher sensitivity to noxious stimuli and more severe inflammatory hyperalgesia in the offspring rats (p18), which could be prevented by the co-administration of DM in the dams

Before carrageenan injection, p18 rats of the morphine group showed a significantly shorter paw withdrawal latency, when compared with that of the control group (7.2 \pm 0.2 sec versus $8.4 \pm 0.4 \mathrm{sec}, \mathrm{n}=8, p<0.001$ ) (Figure 1A). P18 rats of the $\mathrm{M}+\mathrm{DM}$ group showed a similar paw withdrawal latency $(8.1 \pm 0.4 \mathrm{sec}, \mathrm{n}=7)$ to that of the control group. There was no significant difference of the paw withdrawal latency between the DM group $(8.1 \pm 0.4 \mathrm{sec}, \mathrm{n}=10)$ and the control group. These data suggest that chronic morphine administration of the dams caused a higher sensitivity to noxious stimuli in the offspring rats. This effect of morphine could be prevented by the co-administration of DM, whereas DM alone did not cause any significant change in the sensitivity to noxious stimuli.

To examine the effect on inflammatory hyperalgesia, paw withdrawal latency was determined on hour 3, 6, 9, 12, 24, 48 after carrageenan injection (Figure 1B). In the control group, the paw withdrawal latency was significantly decreased to $44.5 \pm 2.7 \%$ and $31.2 \pm 2.8 \%$ of the basal value (value on hour 0) on hour 3 and hour 6 , respectively. This indicates a clear thermal hyperalgesia induced by intra-plantar carrageenan injection in our model. In the morphine group, the paw withdrawal latency was significantly decreased to $33.8 \pm 2.4 \%$ and $24.1 \pm 1.4 \%$ of the basal value on hour 3 and hour 6 , respectively. These latencies decreased to a lower level in comparison with those of the control group ( $p<$ 0.001) (Figure 1B). There was no significant difference between the $M+D M$ group and the control group. These results indicate that the offspring rats from the morphine group showed a more severe inflammatory hyperalgesia, which developed more rapidly compared to controls. However, co-administration of DM with morphine in the dams effectively prevented this adverse effect in the offspring rats. But DM alone did not induce any effect on hyperalgesia.

\section{The quantitative change of NR1/NR2B mRNA and protein expression in the offspring rats from morphine/DM/ (morphine + DM)-treated dams}

In the experiments of Western blots, we found that there was an increase of the expression of NR1 and NR2B subunits of the NMDA receptor within the spinal cord from p14 rats of the morphine group (Figure 2A; $2 \mathrm{C})$. When the quantities of the expression of NR1 and NR2B subunits in the control group were set as $100 \%$, the respective values from the morphine group were $121.1 \pm 1 \%$ in NR1 subunit $(p<0.05)$ and $155 \pm 6.9 \%$ in NR2B subunit $(p<0.001)$ (Figure $2 \mathrm{~B} ; 2 \mathrm{D})$. In the $\mathrm{M}+$ DM group, there was no significant difference from that of the control group in NR1 subunit $(96.5 \pm 27 \%)$, but a significant increase of the NR2B subunit $(122.3 \pm 7.5 \%$, $p<0.05)$ was still observed. Compared with the morphine group, there was a significant lower level of NR2B subunit in the $\mathrm{M}+\mathrm{DM}$ group $(p<0.05)$ (Figure $2 \mathrm{D})$. 

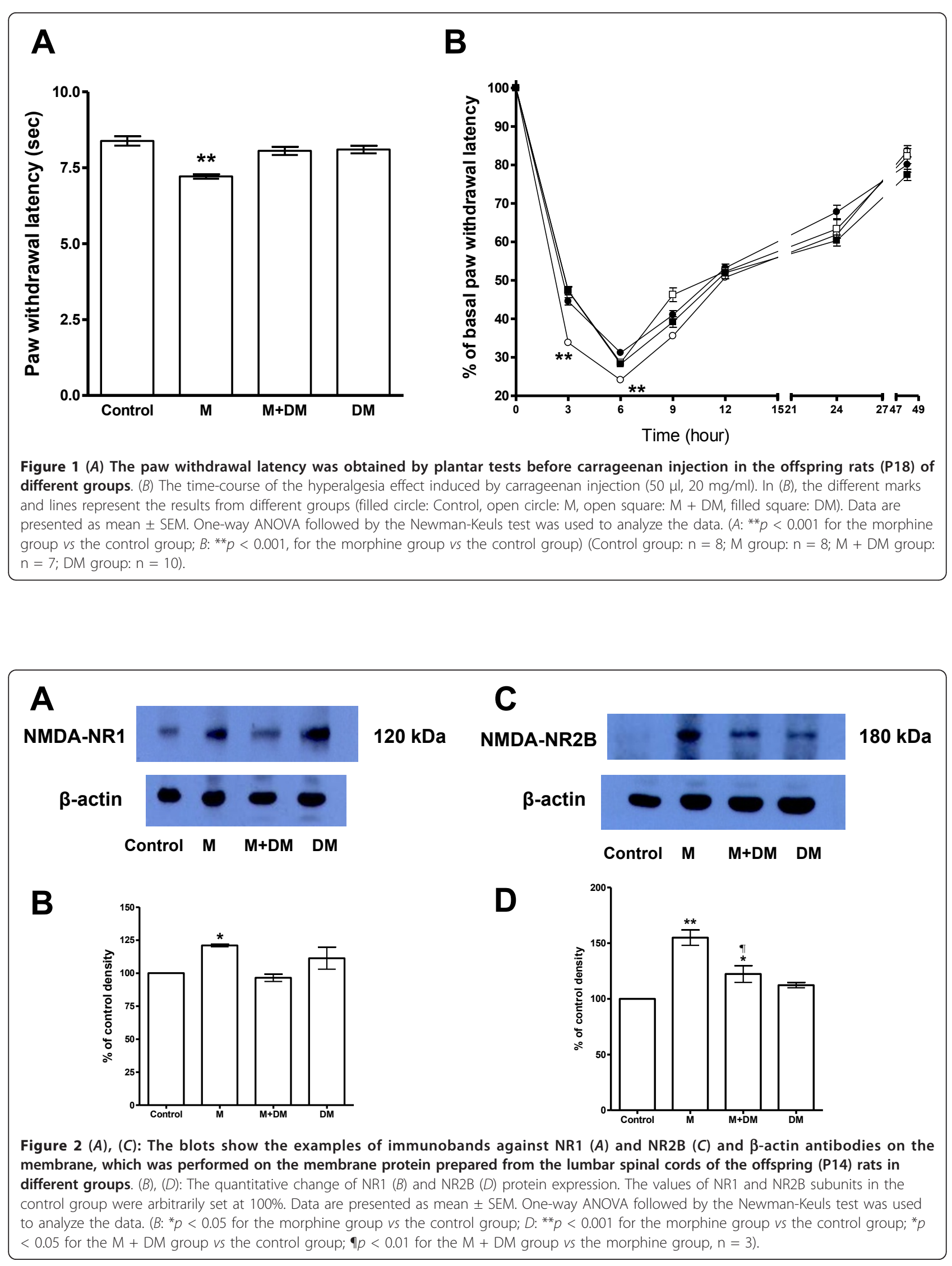
Consistent with the results of Western blots, the levels of mRNA of NR1 and NR2B subunits were also increased in the spinal cords from p14 rats of the morphine group (Figure 3A; $3 \mathrm{C}$ ). When the mRNA values of NR1 and NR2B subunits in the control group were set as $100 \%$, the respective values from the morphine group were significantly increased to $149.3 \pm 16 \%$ in NR1 subunit $(p<0.01)$ and to $132 \pm 7 \%$ in NR2B subunit $(p<0.01)$ (Figure 3B; 3D). In the $\mathrm{M}+\mathrm{DM}$ group, there was no significant difference from that of the control group in both NR1 $(96.6 \pm 1.4 \%)$ and NR2B subunits $(93.6 \pm 6.4 \%)$ (Figure 3B; 3D).

\section{Discussion}

Previously, we found that the co-administration of dextromethorphan with morphine to dam rats throughout pregnancy significantly decreased morphine physical dependence and tolerance in their offspring [5]. In the present study, our results suggested that co-administration of DM with morphine during pregnancy could possibly attenuate the vulnerability to inflammatory hyperalgesia in offspring rats from the dam with chronic morphine exposure. In our experiments, we first observed a lower thermal pain threshold in the offspring rats from morphine-addicted mother. But the pain threshold was found to be as same as that of the control group, when DM was co-administered with morphine in the dams. This implied a higher sensitivity to pain was induced by prenatal exposure to morphine in the dams, which could be diminished by the co-administration of DM. Hovious and Peters first demonstrated that chronic maternal exposure to morphine has a significant effect on the effectiveness of analgesic drugs in the offspring rats [15]. Their results showed decreased response latencies in offspring (p25 and p120) from morphine-treated mother in both tail-flick and hot-plate tests. Although it was more significant in female offspring rats, this report suggested that chronic maternal exposure of morphine could possibly increase the sensitivity to pain in the offspring rats. The following studies also confirmed this
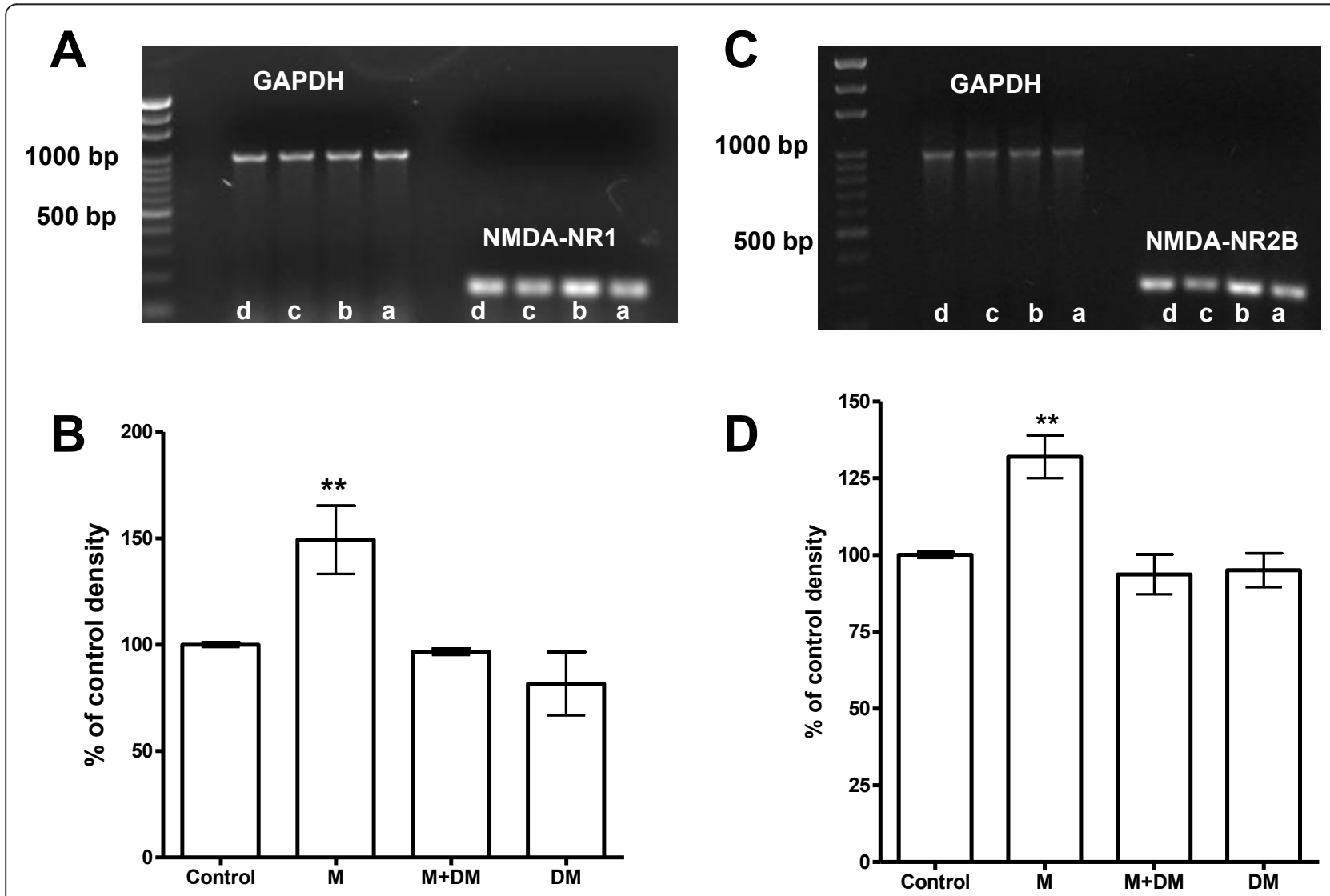

Figure $3(A),(C)$ : An example of RT-PCR results for the levels of mRNA of NMDA receptor NR1 $(A)$ and NR2B $(C)$ subunits and GAPDH $(B)$, (D): The quantitative RT-PCR results for the levels of mRNA of NMDA receptor NR1 (B) and NR2B (D) subunit of the offspring rats (P14) in different groups. In (A), each white letter at the bottom of the lane represents the result from certain group (a: Control, b: M, c: M + DM, d: DM). The values of NR1 and NR2B subunits in the control group were arbitrarily set at 100\%. Data are presented as mean \pm SEM. One-way ANOVA followed by the Newman-Keuls test was used to analyze the data. $\left(B:{ }^{* *} p<0.01\right.$ for the morphine group vs the control group; $D:{ }^{* *} p<0.01$, for the morphine group vs the control group, $\mathrm{n}=3$ ). 
finding of morphine's prenatal effects [16,17]. Although the dosage and schedule of the maternal morphine administration are different, our current results are consistent with these reports. Moreover, we found that maternal co-administration of DM with morphine could prevent the increase of pain sensitivity in the offspring.

In the present study, we further examined the possible effect of morphine's prenatal effect on inflammatory hyperalgesia. Using carrageenan-induced plantar inflammation and plantar test, we observed a more severe hyperalgesia in offspring from morphine-treated dams. Again, this effect could be prevented by the maternal co-administration of DM with morphine. Since maternal treatment of DM itself did not cause any effect on the antinociceptive response and hyperalgesia, DM may be of great therapeutic potential in correlation with the lessening of adverse effects in offspring from morphineaddicted female patients. In view of the age of offspring rats to be tested, we used p18 rats of either sex in the plantar tests. This is also the age of rats showing most significant difference in nociceptive sensitivity between control and prenatal morphine-treated group, which was reported by Zhang and Sweitzer [17]. In this recent report, they found that there was no difference of nociceptive sensitivity between groups at the age over p50.

In search of the possible underlying mechanisms, we examined the level of protein and mRNA of NMDA receptor NR1 and NR2B subunits in the lumbar spinal cords of offspring rats (p14) from different groups. The data showed that the level of NMDA receptor NR1 and NR2B subunits were significantly higher in the morphine group, whereas the maternal co-administration of DM could reverse the effect on NR1 but attenuate the effect on NR2B. Moreover, the prenatal exposure of DM alone did not change the expression of NR1 and NR2B. Interestingly, the mRNA data for NR2B seem to show that the maternal co-administration of DM could totally reverse the effect caused by morphine, which is different from the decrease of NR2B at the protein level. This may be due to the low sensitivity of RT-PCR quantification or the difference between the level of mRNA and protein. The correlation between the NMDA-receptor system and hyperalgesia has been demonstrated since both systemic and intrathecal injections of morphine, specific (MK-801) and nonspecific NMDA-receptor antagonists (DM) could cause a significant reduction of hyperalgesia [18-23]. Moreover, injury-induced hyperalgesia, morphine tolerance, and changes in NR1 mRNA produced by chronic morphine were found to be prevented by the blockade of NMDA receptors in the spinal cord dorsal horn [24-26]. Therefore, the spinal NMDA-receptors were regarded as a functionally important pronociceptive system which was also correlated with hyperalgesia [27]. Although the detailed mechanism of maternal DM to suppress NMDA-receptor expression was unknown, our results provide some possible biochemical evidence in connection with our behavioral findings. Nevertheless, the increase of the expression of NMDA receptor NR1 and NR2B subunits should not be regarded as the sole reason for the higher vulnerability to inflammatory thermal hyperalgesia in prenatal morphine-exposed offspring. Many other biochemical and physical changes could be also involved in the generation of this higher vulnerability. For example, the endogenous opioid peptides and opioid receptors could be changed for their quantities or sensitivities by prenatal morphine exposure. Certainly, the opioid system may contribute to the higher vulnerability to hyperalgesia. This requires further investigations on the possible change of the opioid system. So far, we were only able to conclude that the quantitative change of the NMDA receptor subunits may play a role.

Regarding to the pharmacological target of DM to reduce the adverse effects of prenatal morphine, many behavioral studies revealed that the NMDA receptor antagonism of DM is important for its action to potentiate the antinociceptive effect of morphine in rats $[28,29]$. Although a recent clinical report indicated that MorphiDex (morphine sulfate/dextromethorphan hydrobromide combination) failed to enhance opioid analgesia or reduce tolerance [30], the possible contribution of NMDA receptor blockade by DM could be still of importance in its action to regulate pain. Depending on the dose and the species, NMDA receptor antagonists showed various effects to attenuate pain/nociception in different animal models [31,32]. Therefore, DM may possibly act through the blockade of NMDA receptors to affect morphine-induced higher vulnerability to hyperalgesia in offspring from morphine-treated dams. However, the relevant mechanisms of prenatal DM remain to be tested.

\section{Conclusions}

In summary, the present study provides behavioral and biochemical evidences in neonatal rats passively exposed to morphine throughout embryo stages, which suggest that they could be more susceptible to developing many adverse effects, such as inflammatory hyperalgesia. Therapeutically, DM could reverse this adverse effect caused by prenatal morphine. The current results also implied the possible biological change in the CNS of offspring from morphine-addicted mother in humans. Moreover, the therapeutic potential of DM was further highlighted; especially our recent report also indicated the ability of DM to reduce morphine-induced hyperprolactinemia in female rats at different reproductive stages [33]. 


\section{Acknowledgements}

This study was supported by grants from the National Health Research Institutes (NHRI-99A1-PDCO-0809111), and the National Science Council (NSC 96-2320-B-016-020-MY3), Taipei, Taiwan, R.O.C.

\section{Author details}

'Division of Mental Health and Addiction Medicine, Institute of Population Health Sciences, National Health Research Institutes, 35 Keyan Road, Zhunan, Miaoli County 350, Taiwan. 'Department of Pharmacology, National Defense Medical Center, Taipei, Nei-Hu 114, Taiwan.

\section{Authors' contributions}

CFC carried out the experiments. PLT and EYH conceived of the study, and participated in its design and coordination. All authors read and approved the final manuscript.

\section{Competing interests}

The authors declare that they have no competing interests.

Received: 30 December 2010 Accepted: 23 August 2011 Published: 23 August 2011

\section{References}

1. Johnson JL, Leff M: Children of substance abusers: overview of research findings. Pediatrics 1999, 103:1085-1099

2. Cobrinik RW, Hood RT Jr, Chusid E: The effect of maternal narcotic addiction on the newborn infant; review of literature and report of 22 cases. Pediatrics 1959, 24:288-304

3. Doberczak TM, Kandall SR, Wilets I: Neonatal opiate abstinence syndrome in term and preterm infants. J Pediatr 1991, 118:933-937.

4. Vargas GC, Pildes RS, Vidyasagar D, Keith LG: Effect of maternal heroin addiction on 67 liveborn neonates. Withdrawal symptoms, small body size, and small head circumference were frequent findings. Clin Pediatr (Phila) 1975, 14:751-753.

5. Tao PL, Yeh GC, Su CH, Wu YH: Co-administration of dextromethorphan during pregnancy and throughout lactation significantly decreases the adverse effects associated with chronic morphine administration in rat offspring. Life Sci 2001, 69:2439-2450.

6. Yeh GC, Tao PL, Chen JY, Lai MC, Gao FS, Hu CL: Dextromethorphan attenuates morphine withdrawal syndrome in neonatal rats passively exposed to morphine. Eur J Pharmacol 2002, 453:197-202.

7. Ornoy A, Michailevskaya V, Lukashov I, Bar-Hamburger R, Harel S: The developmental outcome of children born to heroin-dependent mothers, raised at home or adopted. Child Abuse Negl 1996, 20:385-396.

8. Church J, Sawyer D, McLarnon JG: Interactions of dextromethorphan with the N-methyl-D-aspartate receptor-channel complex: single channel recordings. Brain Res 1994, 666:189-194.

9. Hernandez SC, Bertolino M, Xiao Y, Pringle KE, Caruso FS, Kellar KJ: Dextromethorphan and its metabolite dextrophan block alpha3beta4 neuronal nicotinic receptors. J Pharmacol Exp Ther 2000, 293:962-967.

10. Klein M, Musacchio JM: High affinity dextromethorphan binding sites in guinea pig brain. Effect of sigma ligands and other agents. J Pharmacol Exp Ther 1989, 251:207-215.

11. Huang EY, Liu TC, Tao PL: Co-administration of dextromethorphan with morphine attenuates morphine rewarding effect and related dopamine releases at the nucleus accumbens. Naunyn Schmiedebergs Arch Pharmacol 2003, 368:386-392

12. Trujillo KA, Akil H: Inhibition of morphine tolerance and dependence by the NMDA receptor antagonist MK-801. Science 1991, 251:85-87.

13. Wroblewski JT, Danysz W: Modulation of glutamate receptors: molecular mechanisms and functional implications. Annu Rev Pharmacol Toxicol 1989, 29:441-474.

14. Hargreaves K, Dubner R, Brown F, Flores C, Joris J: A new and sensitive method for measuring thermal nociception in cutaneous hyperalgesia. Pain 1988, 32:77-88.

15. Hovious JR, Peters MA: Analgesic effect of opiates in offspring of opiatetreated female rats. Pharmacol Biochem Behav 1984, 21:555-559.

16. Eriksson PS, Ronnback L: Effects of prenatal morphine treatment of rats on mortality, bodyweight and analgesic response in the offspring. Drug Alcohol Depend 1989, 24:187-194.
17. Zhang GH, Sweitzer SM: Neonatal morphine enhances nociception and decreases analgesia in young rats. Brain Res 2008, 1199:82-90.

18. Celerier E, Laulin J, Larcher A, Le Moal M, Simonnet G: Evidence for opiateactivated NMDA processes masking opiate analgesia in rats. Brain Res 1999, 847:18-25.

19. Manning BH, Mao J, Frenk H, Price DD, Mayer DJ: Continuous coadministration of dextromethorphan or MK-801 with morphine: attenuation of morphine dependence and naloxone-reversible attenuation of morphine tolerance. Pain 1996, 67:79-88,

20. Mao J, Price DD, Mayer DJ: Thermal hyperalgesia in association with the development of morphine tolerance in rats: roles of excitatory amino acid receptors and protein kinase C. J Neurosci 1994, 14:2301-2312.

21. Mao J, Price DD, Mayer DJ: Experimental mononeuropathy reduces the antinociceptive effects of morphine: implications for common intracellular mechanisms involved in morphine tolerance and neuropathic pain. Pain 1995, 61:353-364.

22. Mao J, Price DD, Caruso FS, Mayer DJ: Oral administration of dextromethorphan prevents the development of morphine tolerance and dependence in rats. Pain 1996, 67:361-368.

23. Mayer DJ, Mao J, Price DD: The development of morphine tolerance and dependence is associated with translocation of protein kinase C. Pain 1995, 61:365-374.

24. Mao J, Price DD, Mayer DJ: Mechanisms of hyperalgesia and morphine tolerance: a current view of their possible interactions. Pain 1995, 62:259-274.

25. Mao J: Opioid-induced abnormal pain sensitivity: implications in clinical opioid therapy. Pain 2002, 100:213-217.

26. Zhu H, Brodsky M, Gorman AL, Inturrisi CE: Region-specific changes in NMDA receptor mRNA induced by chronic morphine treatment are prevented by the co-administration of the competitive NMDA receptor antagonist LY274614. Brain Res Mol Brain Res 2003, 114:154-162.

27. Koppert W, Schmelz M: The impact of opioid-induced hyperalgesia for postoperative pain. Best Pract Res Clin Anaesthesiol 2007, 21:65-83.

28. Chow LH, Huang EY, Ho ST, Lee TY, Tao PL: Dextromethorphan potentiates morphine antinociception at the spinal level in rats. Can J Anaesth 2004, 51:905-910

29. Chow LH, Huang EY, Ho ST, Tsai SK, Tao PL: Dextromethorphan potentiates morphine-induced antinociception at both spinal and supraspinal sites but is not related to the descending serotoninergic or adrenergic pathways. J Biomed Sci 2004, 11:717-725.

30. Galer BS, Lee D, Ma T, Nagle B, Schlagheck TG: MorphiDex ${ }^{\circledast}$ (morphine sulfate/dextromethorphan hydrobromide) in the treatment of chronic pain: Three multicenter, randomized, double-blind, controlled clinical trials fail to demonstrate enhanced opioid analgesia or reduction in tolerance. Pain 2005, 115:284-295.

31. Bleakman D, Alt A, Nisenbaum ES: Glutamate receptors and pain. Semin Cell Dev Biol 2006, 17:592-604.

32. Brown DG, Krupp JJ: N-methyl-D-aspartate receptor (NMDA) antagonists as potential pain therapeutics. Curr Top Med Chem 2006, 6:749-770.

33. Wu LY, Huang EY, Tao PL: Coadministration of dextromethorphan during pregnancy and throughout lactation prevents morphine-induced hyperprolactinemia in female rats. Fertil Steril 2010, 93:1686-1694.

doi:10.1186/1423-0127-18-64

Cite this article as: Tao et al:: Dextromethorphan attenuated the higher vulnerability to inflammatory thermal hyperalgesia caused by prenatal morphine exposure in rat offspring. Journal of Biomedical Science 2011 18:64. 